\title{
High-Precision Header Flux Control of Ultra-Fast Cooling in Hot Strip Mill By PI Controller
}

\author{
Xudong $\mathrm{Li}^{1}{ }^{\text {a, }}{ }^{\text {, }}$, Fang $\mathrm{Xu}^{2}$, Shuzhi Wang ${ }^{3}$, Bo Gong ${ }^{1}$, Changli Zhang ${ }^{1}$, \\ Ziying Liu ${ }^{1}$ and Fengqin Wang ${ }^{1}$ \\ ${ }^{1}$ Shougang Research Institute of Technology, Beijing 100043, China. \\ ${ }^{2}$ Shougang Jingtang Iron \& Steel Co. Ltd., Tangshan, Yulin 063200, China. \\ ${ }^{3}$ Shougang Qian'an Iron \& Steel Company, Qian'an 064400, China. \\ alixudonggo@163.com
}

Keywords: Hot Strip Rolling; Ultra-fast Cooling; Flux Control; PI Controller.

\begin{abstract}
A new header flux controller of an ultra-fast cooling (UFC) system was investigated during hot strip rolling. The UFC header flux was calibrated by changing the opening rate of the adjusting valve from $0 \%$ to $95 \%$ and, reversely, from $95 \%$ to $0 \%$, in increments of $5 \%$. The results showed that the header flux was rarely influenced by the changing direction of the adjusting valve. Based on the analysis of the flux adjusting, the definition of adjusting-efficiency (A-E) of flux was introduced and the corresponding equation was established. A Proportion-Integral (PI) controller, based on A-E, was employed to implement the closed-loop control of the header flux. The results from the on-site implementation indicated that the flux deviation between target and measured values was less than $\pm 1.0 \mathrm{~m} 3 / \mathrm{h}$.
\end{abstract}

\section{Introduction}

The thermo-metallurgical phase transformations of the steel take place during the cooling process on the run-out table (ROT). The microstructure and mechanical properties of steel are directly related to the cooling process[1-4]. Ultra-fast cooling(UFC) can provide flexible cooling paths in order to achieve the desirable phase transformations, which makes it an appealing selection for the cooling line after rolling $[5,6]$. The key point of the UFC process is to control the UFC delivery temperature (UFC-T) and coiling temperature (CT) accurately, in order to obtain the required strengthening mechanism and microstructure. The UFC-T, as one of the most important processing points during hot strip cooling, is directly controlled by the UFC headers flux. The high-precision header flux control of UFC is considered as the foundation for accurately control of UFC-T.

There are some studies on header flux that are available in the literature[7-10]. The experimental results of these studies show that the header flux influenced the heat transfer coefficient significantly. However, the previous studies were primarily focused on the laws that relate header flux with heat transfer coefficient, under various experimental conditions, while the high precision control method of header flux was rarely involved in hot strip milling. In this study, a fundamental research on UFC header flux was firstly introduced and a PI controller for closed-loop control of header flux was developed. The results were also preliminarily implemented in a production environment.

\section{Fundamental Research on UFC Header Flux}

The UFC system was assembled between the finishing mill and the conventional laminar cooling system as shown in Fig.1. Each UFC unit consists of a curtain header on top of the strip and a spray header at the bottom of the strip. To further eliminate the temperature gradient through the strip thickness and guarantee the target temperature, the flux of each UFC headers can be either controlled independently or tuned in order to coordinate with the rest of the fluxes. 


\subsection{The Components of Water System}

The water system of the UFC unit consists of a constant pressure region, a water delivery pipe region and a ROT region. The constant pressure region consists of a distribution pipe and a water pressure control system. The high-pressure cooling water is pumped into the distribution pipe through the water supply system and discharged from the UFC headers and pressure control system. The main task of the pressure control system is to provide constant pressure cooling water to the UFC header. The water delivery pipes constitute the water delivery region. Each water delivery pipe, connected to the distribution pipe and the UFC header, is equipped with a ON-OFF valve, pneumatic adjusting valve and flow meter to control the header ON-OFF and flux. The objective in this paper was to develop the controller of the adjusting valve and implement the high precision control of the header flux. The UFC headers were mounted on the ROT region, which were the core devices for the implementation of the cooling process of the hot strip after rolling. The schematic of water system for UFC were shown in Fig.2.

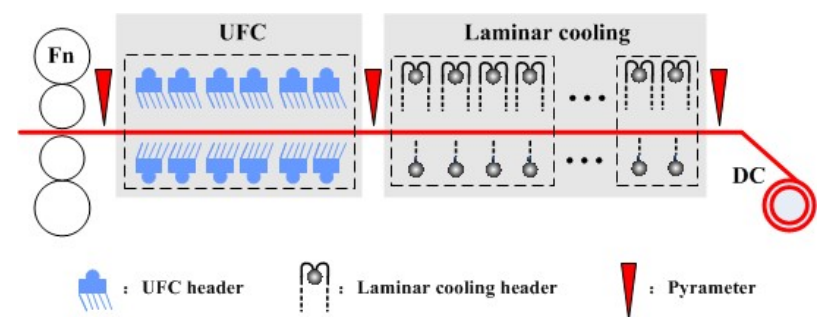

Figure 1. Schematic of installation position of UFC.

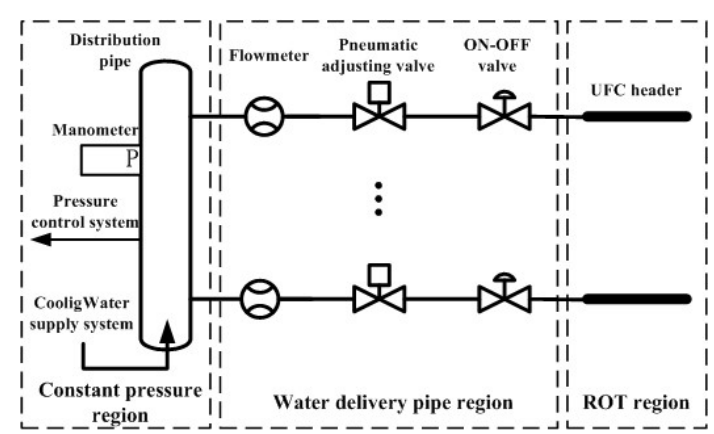

Figure 2. Schematic of water system for UFC.

\subsection{Calibration of Flux Control System}

The UFC header flux was controlled by the adjusting valve, installed on the delivery pipe, under constant water pressure. The UFC header flux was calibrated before the UFC system was put into use. The UFC header flux was calibrated by changing the opening rate of the adjusting valve from $0 \%-95 \%$, in increments of $5 \%$. To ensure the accuracy of the calibration results and eliminate the influence of the changing direction of the adjusting valve, the UFC header flux was also calibrated by performing the reverse procedure and changing the opening rate from $95 \%-0 \%$, at increments of $5 \%$. Table 1 presents the partial calibration results of the header flux under different changing directions of the adjusting valve. It can be observed that the header flux was increased with the increasing of the opening rate of the adjusting valve and the header flux was approximately equal with the same opening rate of the adjusting valve, under the different changing directions of adjusting valve. The calibration results indicated that the header flux was rarely influenced by the changing direction of the adjusting valve. Thus, the influence of changing direction of the adjusting valve on the UFC header flux was ignored during the study.

Table 1. Calibration result of flux under different changing directions of the adjusting valve.

\begin{tabular}{|c|c|c|}
\hline $\begin{array}{c}\text { Opening rate of } \\
\text { adjusting valve (\%) }\end{array}$ & $\begin{array}{l}\text { Header flux under changing direction } \\
\text { of } 0 \%-95 \%\left(\mathrm{~m}^{3} / \mathrm{h}\right)\end{array}$ & $\begin{array}{l}\text { Header flux under changing direction } \\
\text { of } 95 \%-0 \%\left(\mathrm{~m}^{3} / \mathrm{h}\right)\end{array}$ \\
\hline 5 & 8.3 & 8.4 \\
\hline 10 & 29.5 & 30.1 \\
\hline 15 & 68.7 & 68.2 \\
\hline 20 & 99.6 & 99 \\
\hline 25 & 118.7 & 117.2 \\
\hline 30 & 135.8 & 135.2 \\
\hline 50 & 173.7 & 174.3 \\
\hline 70 & 187.9 & 187.8 \\
\hline 95 & 195.3 & 195.4 \\
\hline
\end{tabular}

\subsection{Data Fitting of Calibration Results}

The calibration results, as shown in Table 1, with the characteristics of scattered points, represent the relationship between the opening rate of the adjusting valve and the UFC header flux at a precise 
manner. Due to the discontinuity of the scattered points, a continuous adjustment of the valve opening rate and the UFC header flux curve (D-F curve) was fitted by a 6th rate polynomial, based on the calibration results, and the fitting results took the following form:

$$
f_{\mathrm{F}}=f_{1} \cdot O^{6}+f_{2} \cdot O^{5}+f_{3} \cdot O^{4}+f_{4} \cdot O^{3}+f_{5} \cdot O^{2}+f_{6} \cdot O+f_{7}
$$

where, $\mathrm{fF}$ is the UFC header flux $(\mathrm{m} 3 / \mathrm{h}), \mathrm{fl}-\mathrm{f} 7$ are the D-F curve fitting parameters and $\mathrm{O}$ is the opening rate of the adjusting valve (\%). The D-F curve fitting parameters and the fitting correlation coefficient (R-square) are presented in Table 2.

Table 2. D-F curve fitting parameters and R-square.

$\begin{array}{cccccccc}\boldsymbol{f}_{\mathbf{1}} & \boldsymbol{f}_{\mathbf{2}} & \boldsymbol{f}_{\mathbf{3}} & \boldsymbol{f}_{\mathbf{4}} & \boldsymbol{f}_{\mathbf{5}} & \boldsymbol{f}_{\mathbf{6}} & \boldsymbol{f}_{\mathbf{7}} & \text { R-square } \\ 1.122 \mathrm{E}-8 & -3.567 \mathrm{E}-6 & 4.356 \mathrm{E}-4 & -0.02502 & 0.6166 & -0.4941 & -1.115 & 0.9987\end{array}$

Fig. 3 demonstrates a comparison between the fitting and measured results. It can be observed that the fitting results were in good agreement with the measured results.

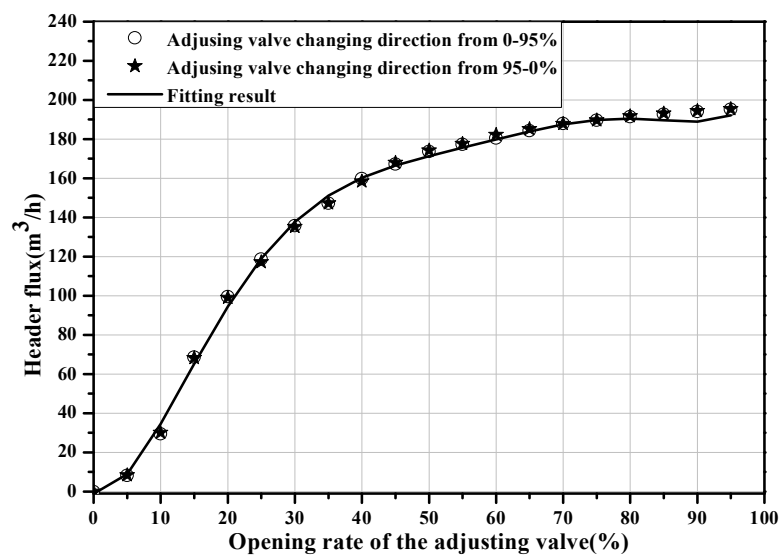

Figure 3. Verification of fitting result.

\section{UFC Header Flux Controller}

\subsection{Definition of Adjusting-efficiency}

In the present work, the adjusting characteristics of the adjusting valve were studied. The definition of " $\frac{\mathrm{d} F}{\mathrm{~d} O}$ " is termed as the adjusting-efficiency (A-E) of the adjusting valve, and the A-E equation could be derived from equation (1). The A-E equation is then converted as follows:

$$
\frac{\mathrm{d} F}{\mathrm{~d} O}=6 f_{1} \cdot O^{5}+5 f_{2} \cdot O^{4}+4 f_{3} \cdot O^{3}+3 f_{4} \cdot O^{2}+2 f_{5} \cdot O+f_{6}
$$

As shown in Fig.4, the A-E curve was obtained by using the A-E equation, which was provided by equation (2). The A-E of the adjusting valve has a nonlinear relationship with the opening rate of the adjusting valve. The A-E also indicated the regulating ability of the adjusting valve under different opening rate. In Fig.4, it can be noticed that the A-E shows the maximum value when the opening rate of the adjusting valve was $14 \%$. 


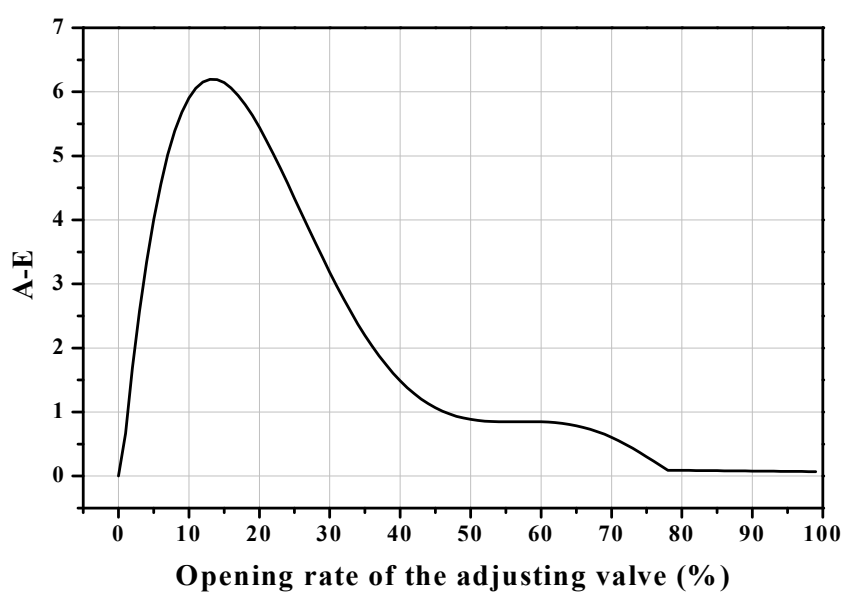

Figure 4. Adjusting-efficiency curve of adjusting valve.

Due to the nonlinear characteristics of the A-E curve, the same adjustment range would exert different adjusting results of the header flux, when the initial opening rate of adjusting valve is different. It was particularly unfavourable to ensure the control precision of header flux and this unfavourable effect was further increased with the deviation of the header flux between the target and measured increment. Hence, the A-E was treated as an important correction factor during the following research.

\subsection{PI Controller Based on Adjusting-efficiency}

The flux controller of the UFC header was able to change the opening rate of the adjusting valve in order for the measured header flux to reach the target value. Before the UFC header was switched on, the open-loop controller was employed to perform the coarse adjustment of the header flux. Based on the calibration results and the target header flux, the initial opening rate was set by the open-loop controller. However, due to the fluctuation of the operating conditions and the influence of the condition of the equipment[11], the deviation of header flux between the targeted and measured was unavoidable. During the operation of the UFC header, the closed-loop controller was applied. In the closed-loop process, a new PI controller, based on A-E, was developed. The main purpose of the newly developed PI controller was to rapidly eliminate the deviation, caused by the open-loop, and accurately control the header flux. The equation of the PI controller is as follows:

$$
u(k)=F_{\mathrm{P}} \cdot e(k)+F_{\mathrm{I}} \cdot{ }_{0}^{i} e(k)
$$

where, $\mathrm{u}(\mathrm{k})$ is the correction of the opening rate of the adjusting valve (\%), FP, FI are the correction coefficients based on the A-E for the P and I controller, respectively, $\mathrm{e}(\mathrm{k})$ is the deviation of the header flux between the target and measured values $(\mathrm{m} 3 / \mathrm{h})$, and $\mathrm{i}$ is the integration time (s).

Fig. 5 presents a schematic diagram of the newly developed PI controller. The e(k) is calculated when the deviation controller receives the measured flux values. The FP and FI could obtained according to the real-time opening rate of the adjusting valve in the parameters tables. The correction of the opening rate of the adjusting valve was calculated by the equation (3) then and sent to the actuator to perform the closed-loop control for the header flux.

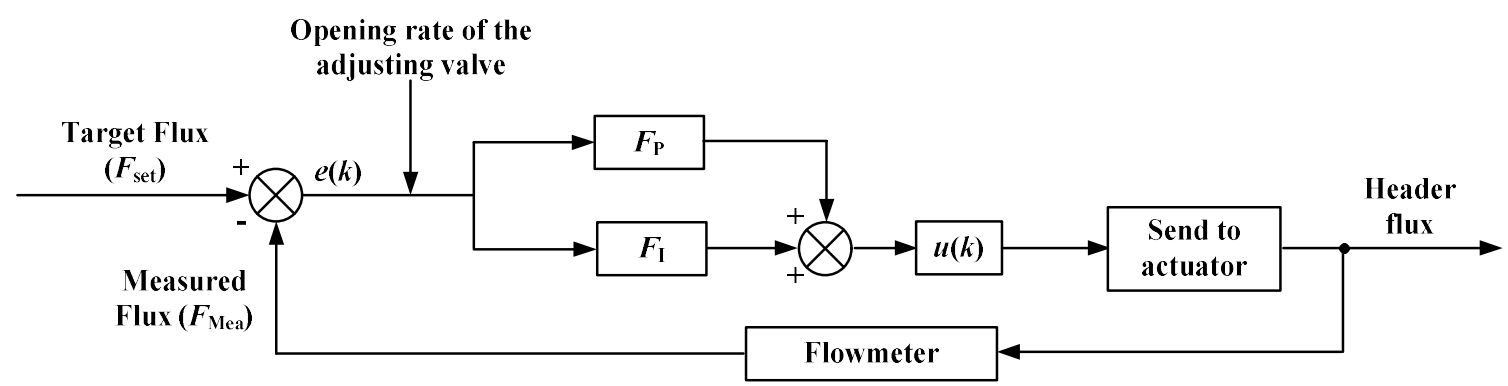

Figure 5. Schematic diagram of the newly developed PI controller. 


\section{Field Application}

The newly developed PI controller, based on A-E, was successfully applied to a hot strip plant. The stable control results of the header flux were obtained. Fig.6 shows the measured flux of a UFC header in a production line.

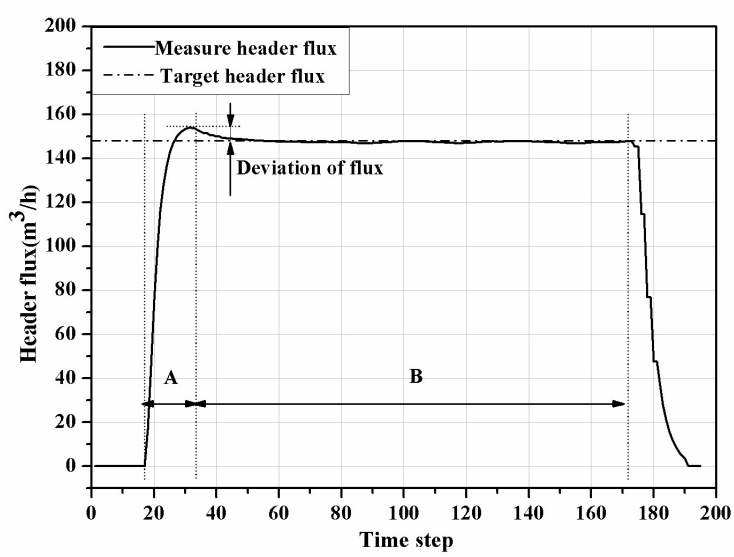

Figure 6. Flux control result of the newly developed PI controller.

As shown in the Fig.6, at the left part of section A, the increasing process of header flux can be observed and then the flux tends to stabilize under an open-loop controller; the section B represents the closed-loop process. The target UFC header flux was $148.0 \mathrm{~m} 3 / \mathrm{h}$ and the deviation of flux was up to $5 \mathrm{~m} 3 / \mathrm{h}$ before the closed-loop controller was put into operation. At the beginning of the section $\mathrm{B}$, it can be observed that the deviation of the flux, caused by open-loop process, was rapidly eliminated by the closed-loop process, due to the newly developed PI controller. The flux deviation between the target and measured was less than $\pm 1.0 \mathrm{~m} 3 / \mathrm{h}$ during the UFC header constant operation.

\section{Conclusion}

The flux of the UFC header was calibrated by changing the opening rate of adjusting valve from $0 \%$ to $95 \%$ and, reversely, from $95 \%$ to $0 \%$, in increments of $5 \%$. The calibration results show that the flux of the header was rarely influenced by the changing direction of the adjusting valve. Based on the analysis of the flux adjusting, the adjusting-efficiency (A-E) of flux was introduced and the corresponding equation was established. Based on the A-E, the PI controller was employed to realize the closed-loop control of header flux. The newly developed controller for the UFC header flux control was successfully applied to a hot strip plant. The flux deviation between the target and measured values was less than $\pm 1.0 \mathrm{~m} 3 / \mathrm{h}$ during the UFC header constant operation. A high efficient and stable control effect was achieved by the newly developed controller.

\section{References}

[1]. B. Wang and Z. D. Wang, Metall Mater Trans A 46A, 2834-2843 (2015).

[2]. J. Chen and M. Y. Lv, Isij Int 54, 2926-2932 (2014).

[3]. S. Tang and Z. Y. Liu, Mater Sci Eng A 580, 257-265 (2013).

[4]. L. G. Peng and X. H. Liu, J Cent South Univ 21, 43-49 (2014).

[5]. Z. L. Li and H. J. Li, Steel Res Int 86, 478-488 (2015).

[6]. T. L. Fu and Z. D. Wang, Appl Therm Eng 78, 500-506 (2015).

[7]. F. C. XuandM. S. Gadala, Int J Heat Mass Tran 49, 3785-3799 (2006).

[8]. P. Bhattacharya and A. N. Samanta, Int J Therm Sci 48, 1741-1747 (2009). 
[9]. M. Gradeck and A. Kouachi, Int J Heat Mass Tran 54, 5527-5539 (2011).

[10]. J. Wendelstorf and K. H. Spitzer, Int J Heat Mass Tran 51, 4902-4910 (2008).

[11]. H. J. Li and L. G. Li, Steel Res Int 86, 1225-1233 (2015). 